Abstract 19 Table 1 Difference and percent change needed in CLASI-A scores to predict meaningful improvement in Skindex-29 subscales (Emotions and Symptoms) in patients with a range of initial CLASI-A scores

\begin{tabular}{|c|c|c|c|}
\hline \multicolumn{2}{|c|}{ Initial CLASI-A $\geq 8$} & \multirow{2}{*}{$\begin{array}{l}\text { Percent Change } \\
\text { in CLASI-A }\end{array}$} & \multirow[t]{2}{*}{ p-value } \\
\hline$n=90$ & Skindex-29 Subscale & & \\
\hline Emotions & & 42.06 & $<0.0001$ \\
\hline Symptoms & & 30.97 & $<0.0001$ \\
\hline \multicolumn{4}{|c|}{ Mild Disease Activity (CLASI-A 4-9) } \\
\hline$n=49$ & Skindex-29 Subscale & & \\
\hline Emotions & & 75.04 & 0.0004 \\
\hline Symptoms & & 110.0 & 0.0510 \\
\hline \multicolumn{4}{|c|}{ Moderate Disease Activity (CLASI-A 10-20) } \\
\hline$n=46$ & Skindex-29 Subscale & & \\
\hline Emotions & & 49.11 & 0.0050 \\
\hline Symptoms & & 33.96 & $<0.0001$ \\
\hline \multicolumn{4}{|c|}{ Severe Disease Activity (CLASI-A>20) } \\
\hline$n=31$ & Skindex-29 Subscale & & \\
\hline Emotions & & 25.42 & 0.0010 \\
\hline Symptoms & & 18.90 & 0.0080 \\
\hline Initial CLA & $\mathrm{I}-\mathrm{A} \geq 8$ & Difference in CLASI-A & p-value \\
\hline$n=90$ & Skindex-29 Subscale & & \\
\hline Emotions & & 6.872 & $<0.0001$ \\
\hline Symptoms & & 5.107 & $<0.0001$ \\
\hline \multicolumn{4}{|c|}{ Mild Disease Activity (CLASI-A 4-9) } \\
\hline$n=49$ & Skindex-29 Subscale & & \\
\hline Emotions & & 4.630 & 0.0006 \\
\hline Symptoms & & 5.722 & 0.0235 \\
\hline \multicolumn{4}{|c|}{ Moderate Disease Activity (CLASI-A 10-20) } \\
\hline$n=46$ & Skindex-29 Subscale & & \\
\hline Emotions & & 7.415 & 0.0067 \\
\hline Symptoms & & 5.051 & $<0.0001$ \\
\hline \multicolumn{4}{|c|}{ Severe Disease Activity (CLASI-A>20) } \\
\hline$n=31$ & Skindex-29 Subscale & & \\
\hline Emotions & & 6.541 & 0.0002 \\
\hline Symptoms & & 4.426 & 0.0011 \\
\hline
\end{tabular}

disease activity, defined by the Cutaneous Lupus Disease Area and Severity Index (CLASI) score for activity (CLASI-A), in patients with an initial CLASI-A 10. We aim to define the degree of improvement in disease activity needed to have a meaningful impact on QoL, an important variable in the design and interpretation of future clinical trials.

Methods This study included 126 patients seen at the Autoimmune Skin Disease Clinic at the Hospital of the University of Pennsylvania who participate in a longitudinal research database. Patients with mild, moderate or severe initial CLASI-A were analyzed separately, with further stratification of patients with mild initial activity. A linear regression model was used to calculate the percent change and difference needed in CLASI-A to have an important impact on QoL, defined as a 9.38-point and a 7.37-point improvement in the Emotions and Symptoms subscales of Skindex-29, respectively.

Results In patients with an initial CLASI-A 8, an improvement of $42.1 \%$ or 7 -points and an improvement of $31.0 \%$ or 5 points in disease activity is associated with a meaningful improvement in the Emotions and the Symptoms subscales, respectively. For both subscales, patients with increasingly severe initial disease required a smaller percent change in CLASI-A to predict a meaningful change in QoL (table 1).

Conclusions We find that using a CLASI-A 8 for trial entry allows for the inclusion of patients with milder disease for whom improvement of CLASI-A by 50\% results in a meaningful impact on QoL, as determined by the Emotions and Symptoms subscales of Skindex-29. In patients with CLASI-A 8 , a decrease in activity by seven and five-points is not only a clinically significant improvement but also indicates a meaningful impact on the Emotions and Symptoms subscales, respectively. For trials enrolling patients with CLASI-A 20, we recommend stratifying patients by disease severity, as a smaller percent change in activity in patients with severe disease can predict meaningful improvement in QoL. Our findings establish appropriate trial endpoints by determining clinically significant change in disease activity associated with meaningful changes in patients QoL.

Funding Source(s): NIH/NIAMS 1R01AR071653 - 01A1

\section{ANTI-NEUTROPHIL CYTOPLASMIC ANTIBODIES IN LUPUS NEPHRITIS}

${ }^{1}$ May Choi ${ }^{*},{ }^{2}$ Ann E Clarke, ${ }^{3}$ Alex Chin, ${ }^{4}$ Michelle Jung, ${ }^{2}$ Claire Barber, ${ }^{4}$ Marvin Fritzler. ${ }^{1}$ Cumming School of Medicine, University of Calgary; ${ }^{2}$ Division of Rheumatology, Cumming School of Medicine, University of Calgary; ${ }^{3}$ University of Calgary, Calgary Laboratory Services; ${ }^{4}$ University of Calgary

\subsection{6/lupus-2019-Ism.20}

Background Anti-myeloperoxidase (MPO) antibodies have been shown to predict the development of proliferative lupus nephritis (LN) suggesting anti-neutrophil cytoplasmic antibodies (ANCA) may have a pathogenic and prognostic role in LN. This study compared the type of LN, renal function, and systemic lupus erythematosus (SLE)-related and antiphospholipid autoantibodies between LN patients who were ANCA (antiproteinase 3 (PR3) and anti-MPO antibodies) positive and negative.

Methods Patients fulfilling the ACR or SLICC Classification Criteria for SLE were enrolled in a local cohort. We retrospectively identified patients with Class 2, 3, 4, or $5 \mathrm{LN}$ on renal biopsy who also had an ANCA, plasma creatinine, and urine protein creatinine ratio (UPCR) at time of biopsy. ANCA by IIF was performed on ethanol and formalin-fixed polymorphonuclear leukocytes and a HEp-2 cell biochip (EuroPattern, Euroimmun $\mathrm{GmbH}$, Luebeck, Germany) while antibodies to MPO and PR3 were determined by multiplex immunoassay (Bio-Rad, Hercules, CA: BioPlex 2200, cutoff $\geq 2 \mathrm{KEU} / \mathrm{L})$. Using sera collected at enrollment, SLErelated autoantibodies (dsDNA, Sm, U1RNP, Sm, Ro52/ TRIM21, Ro60/SSA, SS-B/La, Scl-70, Jo-1, RiboP, PCNA, PM/ $\mathrm{Scl}$ ) were performed by laser bead immunoassay (Euroimmune), lupus anticoagulant by tissue thromboplastin inhibition test and dilute Russell viper venom time, and anti-cardiolipin IgG and anti-2 glycoprotein-1 IgG by ELISA. Comparisons were performed with Fishers exact or Mann-Whitney U.

Results 23 SLE patients with LN were included; $82.6 \%$ were female. Most patients $(20 / 23,87.0 \%)$ were ANCA positive by IIF while only $5 / 23(21.7 \%)$ had antibodies to MPO $(3 / 23$, $13.0 \%)$ or both $\mathrm{MPO}$ and PR3 (2/23, 8.7\%). Anti-MPO/PR3 positive patients had p-ANCA $(2 / 5,40 \%)$ or an atypical pattern $(3 / 5,60 \%)$ on ANCA IIF. When comparing anti-MPO/ PR3 positive (5) to negative (18) patients, there was no difference in LN class, creatinine, UPCR, or the presence of SLErelated autoantibodies. Anti-cardiolipin IgG antibodies were more common in anti-MPO/PR3 positive patients $(60.0 \%$ vs $5.6 \%, \mathrm{p}=0.021)$, while a nuclear pattern on ANCA IIF was more common in anti-MPO/PR3 negative patients $(55.6 \%$ vs. 
$0 \%, \mathrm{p}=0.046)$. Of note, three of the five $\mathrm{LN}$ patients with cerebrovascular accidents or venous thrombosis were antiMPO/PR3 positive.

Conclusions Positive ANCA by IIF was common in LN, however, only a fifth of LN patients had anti-MPO/PR3 antibodies which were associated with anti-cardiolipin $\operatorname{IgG}$ antibodies. Anti-MPO/PR3 negative patients were more likely to have a positive ANCA IIF with nuclear staining. We are currently comparing ANCA positivity between SLE patients with and without LN.

Funding Source(s): The Arthritis Society Chair in Rheumatic Diseases at the Cumming School of Medicine, University of Calgary

\section{IS LUPUS NEPHRITIS ONSET DELAYED IN OLDER CAUCASIAN FEMALES WITH LESS AGGRESSIVE PATHOLOGY?}

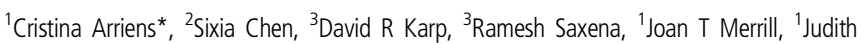
A James. 'Oklahoma Medical Research Foundation; ${ }^{2}$ University of Oklahoma Health Sciences Center; ${ }^{3}$ UT Southwestern Medical Center

\subsection{6/lupus-2019-Ism.21}

Background Lupus Nephritis (LN) usually presents within five years of SLE diagnosis. Male gender, Hispanic and African American race, and childhood-onset SLE portend greater risk of early nephritis. However, patients with late-occurring nephritis are poorly characterized. Since proliferative nephritis is a more aggressive class of nephritis than membranous and mesangial, we hypothesized that female gender, Caucasian race, adult-onset SLE, and membranous pathology would be associated with a later onset of nephritis.
Methods A single large urban medical center was the source for retrospective record review of patients who underwent kidney biopsy between 1999 and 2015. Patients with biopsyproven LN were evaluated to determine whether gender, race, age at SLE diagnosis, age at first biopsy, or histopathologic classification were associated with time between SLE diagnosis and first renal biopsy.

Results 630 patients were screened. After elimination of duplicates, those without initial biopsy, biopsy findings other than LN, and unknown date of SLE diagnosis, 293 subjects had adequate data available for analysis. Those with late onset SLE, diagnosis after age 50, were excluded from further analysis due to known differences in this subgroup. The remaining 278 patients had characteristics shown in table 1 . Twenty-nine percent of patients in this cohort developed nephritis more than 5 years after initial SLE diagnosis. Multivariable linear regression identified greater age at first biopsy and race as the strongest predictors of time between SLE diagnosis and first renal biopsy. Hispanic patients were more likely to present with LN earlier in their course and Asian patients were more likely to present later, with African American and Caucasian groups between them. The model accounts for 27\% of the variability in the time elapsed between diagnosis and biopsy. Histopathologic class did not make a significant contribution, and proliferative nephritis was noted in similar numbers of patients who developed LN after 5 years and those in the early presenting group.

Conclusions Surprisingly, more than a quarter of known LN patients at this large US center present after 5 years of SLE, higher than expected based on low numbers of late onset nephritis in other cohorts. This appears to be more common in Caucasian and Asian subgroups. Older patients are more likely to develop nephritis later, but aggressive histopathology is not less likely in later presentations. The data underscore

\begin{tabular}{|c|c|c|c|c|c|}
\hline Characteristic & $\begin{array}{c}\text { Total } \\
(n=278)\end{array}$ & $\begin{array}{l}\leq 5 \text { Years } \\
(n=197)\end{array}$ & $\begin{array}{c}>5 \text { Years } \\
(n=81)\end{array}$ & $\begin{array}{c}>10 \text { Years } \\
(n=45)\end{array}$ & $\begin{array}{c}>15 \text { Years } \\
(n=22)\end{array}$ \\
\hline Female (n, \%) & $242(87.05)$ & $167(84.77)$ & $75(92.59)$ & $42(93.33)$ & $21(95.45)$ \\
\hline $\begin{array}{l}\text { Race/Ethnicity (n, \%) } \\
\text { Asian } \\
\text { White/Caucasian } \\
\text { Black/African American } \\
\text { Hispanic/Latino }\end{array}$ & $\begin{array}{c}18(6.47) \\
27(9.71) \\
121(43.53) \\
112(40.29)\end{array}$ & $\begin{array}{c}9(4.57) \\
17(8.63) \\
84(41.12) \\
90(45.69)\end{array}$ & $\begin{array}{r}9(11.11) \\
10(12.35) \\
40(49.38) \\
22(27.16)\end{array}$ & $\begin{array}{r}5(11.11) \\
7(15.56) \\
21(46.67) \\
12(26.67)\end{array}$ & $\begin{array}{c}2(9.09) \\
5(22.73) \\
12(54.55) \\
3(13.64)\end{array}$ \\
\hline Age at SLE (median, IQR) & $\begin{array}{l}25.45 \\
{[19.12,33.20]}\end{array}$ & $\begin{array}{l}25.64 \\
{[19.49,35.03]}\end{array}$ & $\begin{array}{l}25.16 \\
{[18.31,30.22]}\end{array}$ & $\begin{array}{l}24.36 \\
{[18.12,29.34]}\end{array}$ & $\begin{array}{l}24.5 \\
{[19.16,27.03]}\end{array}$ \\
\hline Age at Lupus Nephritis (median, IQR) & $\begin{array}{l}29.78 \\
{[22.26,38.43]}\end{array}$ & $\begin{array}{l}26.88 \\
{[20.49 .36 .49]}\end{array}$ & $\begin{array}{l}36.24 \\
{[30.02,44.78]}\end{array}$ & $\begin{array}{l}42.34 \\
{[33.06,48.88]}\end{array}$ & $\begin{array}{l}45.38 \\
{[38.34,51.75]}\end{array}$ \\
\hline \multicolumn{6}{|l|}{ Histopathologic Class (n, \%) } \\
\hline II & $33(11.87)$ & $24(12.18)$ & $9(11.11)$ & $6(13.33)$ & $5(22.73)$ \\
\hline III & $65(23.38)$ & $45(22.84)$ & $20(24.69)$ & $14(31.11)$ & $7(31.82)$ \\
\hline $\mathrm{III+V}$ & $41(14.75)$ & $25(12.69)$ & $16(19.75)$ & $5(11.11)$ & $1(4.55)$ \\
\hline IV & $64(23.02)$ & $49(24.87)$ & $15(18.52)$ & $7(15.56)$ & $3(13.64)$ \\
\hline$I V+V$ & $24(8.63)$ & $16(8.12)$ & $8(9.88)$ & $4(8.89)$ & $1(4.55)$ \\
\hline V & $51(18.35)$ & $38(19.29)$ & $13(16.05)$ & $9(20.00)$ & $5(22.73)$ \\
\hline Proliferative (III, III+V, IV, IV+V) & $194(69.78)$ & $135(68.53)$ & $59(72.84)$ & $30(66.67)$ & $12(54.55)$ \\
\hline
\end{tabular}

The initial group is the total cohort, followed by the cohort divided into $<5$ years between SLE and first LN biopsy and $>5$ years. All $>10$ years patients are also included within the $>5$ years group, and $>15$ years patients are also in $>5$ years and $>10$ years groups. Groups are presented for illustrative purposes only. Time between SLE and biopsy was analyzed as a continuous outcome variable to prevent unnecessary loss of power. 